\title{
Averaged Description of Flow (Steady and Transient) and Nonreactive Solute Transport in Random Porous Media
}

\author{
Mark Shvidler and Kenzi Karasaki
}

\author{
Earth Sciences Division, Lawrence Berkeley National Laboratory, 1 Cyclotron Rd, MS \\ 90-1116, Berkeley, CA 94720; Mshvidler@lbl.gov, kkarasaki@lbl.gov
}

\begin{abstract}
In previous papers (Shvidler and Karasaki, 1999, 2001, 2005, and 2008) we presented and analyzed an approach for finding the general forms of exactly averaged equations of flow and transport in porous media. We studied systems of basic equations for steady flow with sources in unbounded domains with stochastically homogeneous conductivity fields. A brief analysis of exactly averaged equations of nonsteady flow and nonreactive solute transport was also presented. At the core of this approach is the existence of appropriate random Green's functions. For example, we showed that in the case of a 3-dimensional unbounded domain the existence of appropriate random Green's functions is sufficient for finding the exact nonlocal averaged equations for flow velocity using the operator with a unique kernel-vector. Examination of random fields with global symmetry (isotropy, transversal isotropy and orthotropy) makes it possible to describe significantly different types of averaged equations with nonlocal unique operators.

It is evident that the existence of random Green's functions for physical linear processes is equivalent to assuming the existence of some linear random operators for appropriate stochastic equations. If we restricted ourselves to this assumption only, as we have done in this paper, we can study the processes in any dimensional bounded or unbounded fields and in addition, cases in which the random fields of conductivity and porosity are stochastically nonhomogeneous, nonglobally symmetrical, etc..

It is clear that examining more general cases involves significant difficulty and constricts the analysis of structural types for the processes being studied. Nevertheless, we show that we obtain the essential information regarding averaged equations for steady and transient flow, as well as for solute transport.
\end{abstract}

Key words: heterogeneous porous media, random, averaging, nonlocal, flow, transient flow, nonreactive solute transport, operator approach

\section{INTRODUCTION}

The operator approach for analyzing some linear physical fields, such as problems of elasticity, and in particular problems of flow in porous media, has been applied in many earlier studies (Finkelberg, 1964; Shermergor,1977; Shvidler, 1985, 1993; Neuman and Orr, 1993; Tartakovsky and Neuman, 1998; Indelman, 2002,etc).

As we outlined in Shvidler and Karasaki(2008) for example in the case of steady flow with sources many investigators a priori assume that in the general case there is linear relationship between the mean flow velocity vector and the gradient of the mean head or pressure. They have used this assumption to exclude the nonrandom density of the source function, for which one needs 
to utilize an inverse vector-operator. As is evident in the general case a unique inverse vectoroperator does not exist. We showed that this approach in general leads to an ill-posed problem.

In addition to the problem discussed above, there is another source of error that occurs when the solution converted by the Fourier or Fourier-Laplace transforms of integral flow-equations is solved directly by perturbation methods (Indelman and Abramovich, 1994; Indelman, 1996). Assuming that the normalized converted fluctuations of the conductivity function are relatively small, investigators have used the Liouville-Neuman iteration procedure for expanding the solution of the integral equation in a series of powers of this parameter. We have previously noted (Shvidler and Karasaki, 2008) that these perturbation series always diverge.

Next, each averaged term of this series includes the integral of the product of appropriate mixed moment for the converted fluctuation of the conductivity and the converted nonrandom function of the source intensity. Using some unproven formulas -A3 in Indelman and Abramovich, 1994 and A2 in Indelman, 1996, the authors stated that for any conductivity random field and any term of the perturbation series ( for all approximation orders) are proportional to the nonrandom converted function of sources. This manipulation is repeated with the converted flow-velocity perturbation series, which leads to the final manipulation of excluding the converted function of sources from the appropriate order series of the converted head gradients and the converted flowvelocity. It is evident that using this approach, one must utilize an inverse converted vectorfunction, which is, as discussed above, an ill-posed problem.

Furthermore, a simple analysis shows that the cited formulas (A2 and A3) are correct only in two cases: (1) the second order moment for any stochastically homogeneous random fields and (2) when the random conductivity function is a Gaussian field. In the latter case the moments of $2 n$ orders can be written as the sum of (2n-1)!! terms, each of which is the product of the second order different points' moments, and in all the terms we have any possible combinations of pairs. The odd order moments are zero. In addition, noteit that assuming that the conductivity or its converted fluctuation is a Gaussian function is only a crude approximation of real fields. Thus the manipulation that excludes the converted function of sources from any order perturbation series is incorrect.

We need to emphasize that when averaging stochastic equations of flow and transport, it is important how we average the equation for the flow velocity or solute flux. As we proved and discussed in Shvidler and Karasaki (2008), deriving averaged equations like Darcy's with the mean flow velocity linearly dependent on the mean gradient of head or gradient of pressure, leads in general to non-unique characteristics for these models.

It is evident that in linear relations between the averaged flow velocity and the averaged gradient of head the density of sources or sinks $f(\mathbf{x})$ or $f(\mathbf{x}, t)$ need not be included in the explicit form. Some methods of excluding these functions lead to different results.

Another possible approach is to study some fictitious and relatively simple process in the so-called matching body. For example, we can study flow in homogeneous and isotropic nonrandom porous media, while setting the density of flow and sinks in this matching body identical to that in the original heterogeneous body. In this case it is easy to study the fictitious process in the matching body and obtain the relevant explicit nonrandom flow velocity and head distributions.

Then, comparing the continuity equations for real processes in heterogeneous media to those in the matching body, we obtain the relationship between the desired head function and the known fictitious head function. The next step consists of calculating and averaging the flow velocity in real space. Of course the final relations for flow velocity include some functionals of preassigned fictitious processes. 
Many applications of this approach can be found in dealing with similar problems, for example in the theory of elasticity (Hashin and Strikman, 1962, Shermergor, 1979) and in porous media flow problems set forth by Shvidler (1985).

The well known series of publications by S.P. Neuman et al., starting with Neuman and Orr (1993), developed some modifications to this approach. For example Tartakovsky and Neuman (1998) analyzed transient flow in unbounded stochastically homogeneous fields, deriving nonlocal equations (Equation 32 within that paper) for the mean flow velocity, which are-a convolution of kernel-tensor function and the gradient of head function. It is easy to see that the kernel-function is non-unique.

In addition, as we proved in Shvidler and Karasaki (2005 and 2008) the exact equation for flowvelocity in the general case of a stochastically homogeneous field is the convolution of unique kernel-vector and the head-function. However, in some cases involving global symmetry (isotropy, transversal isotropy or orthotropy) the convolution reduced to a unique and appropriate symmetric tensor and vector of head gradient (Shvidler and Karasaki, 2008).

Note that as in our previous publications (Shvidler and Karasaki, 1999, 2001, 2005, 2008) here we utilize appropriate Green's functions and some of their properties. In all the cases examined below (flow, transient flow and transport of solute) we analyze processes in both unbounded and bounded domains. For calculating the averaged flow velocity we use the resulting relations between local random head and averaged head, and for calculating the averaged solute flux the local random concentration of solute and the averaged concentration of solute. In this way we find the forms of the exact averaged equations without directly solving the stochastic equations or using any assumptions regarding small parameters.

By analyzing the processes in fields with fast oscillating parameters many effective approaches have been developed, one of which is the so-called homogenization. Similar variants have been used to study linear as well as some nonlinear processes in random and periodical structures (see, for example, Bakhvalov and Panasenko 1989; Zhikov et al.1994; Hornung 1997).

\section{Steady Flow with Sources and Sinks}

We consider a steady flow with sources and sinks that are locally or continuously distributed in a porous, single-connected, heterogeneous, d-dimensional, bounded domain. The local condition of flow continuity and Darcy's law, the boundary condition are given by the following equations:

$$
\begin{aligned}
& \nabla \mathbf{v}(\mathbf{x})=f(\mathbf{x}), \mathbf{v}(\mathbf{x})=-\boldsymbol{\sigma}(\mathbf{x}) \nabla u(\mathbf{x}), \quad \mathbf{x} \in \Omega \\
& u(\mathbf{x})=u_{0}=\text { const }, \quad \mathbf{x} \in \partial \Omega
\end{aligned}
$$

Here, $\mathbf{x}=\left(x_{1}, \ldots, x_{d}\right)$ is a d-dimensional vector; $\mathbf{v}(\mathbf{x})$ is the Darcy's velocity vector; $\boldsymbol{\sigma}(\mathbf{x})$ is the random second rank conductivity tensor, symmetric by subscript, positive definite and limited; and $u(\mathbf{x})$ is the reduced pressure (or hydraulic head). The function $f(\mathbf{x})$ is the given density of sources and sinks, which is an integrable and compactly supported function or a distribution with bounded support. In this case, $q=\int_{\Omega} f(\mathbf{x}) d x^{d}$ is finite. The function $f(\mathbf{x})$ and the constant $u_{0}$ are nonrandom.

Equations (2.1) and (2.2) determine the relationsips among the random functions $u(\mathbf{x})$ and $\mathbf{v}(\mathbf{x})$, non-random function $f(\mathbf{x})$ and constant $u_{0}$. Our goal is to find the general form of the 
closed system of relationships between the averaged fields $U(\mathbf{x})=\langle u(\mathbf{x})\rangle, \mathbf{V}(\mathbf{x})=\langle\mathbf{v}(\mathbf{x})\rangle$ and the given function $f(\mathbf{x})$ and constant $u_{0}$.

Combining now Equations (2.1) and (2.2) we have for each realization of the conductivity field the differential elliptic equation and boundary condition for the scalar function $u^{\prime}(\mathbf{x})=u(\mathbf{x})-u_{0}$

$-\nabla\left[\boldsymbol{\sigma}(\mathbf{x}) \nabla u^{\prime}(\mathbf{x})\right]=f(\mathbf{x}), \mathbf{x} \in \Omega$

$u^{\prime}(\mathbf{x})=0, \mathbf{x} \in \partial \Omega$

The $u^{\prime}(\mathbf{x})$-the solution of the system (2.3)-(2.4), exists and is unique. In some cases $u^{\prime}(\mathbf{x})$ can be the generalized function. For this solution we have the condition:

$M u^{\prime}=f$

where $M=-\nabla \boldsymbol{\sigma}(\mathbf{x}) \nabla$ is the random operator that for almost all realizations of conductivity tensor $\boldsymbol{\sigma}(\mathbf{x})$ maps the random spaces of the mentioned solutions - functions $u^{\prime}(\mathbf{x})$ onto the nonrandom space of functions $f(\mathbf{x})$.

In accordance with the Green's formula we have the unique solutions of system (2.3)- (2.4) ( see for example Koshlyakov et al, 1964 )

$u^{\prime}(\mathbf{x})=\int_{\Omega} g(\mathbf{x}, \mathbf{y}) f(\mathbf{y}) d y^{d}$

Here the kernel $g(\mathbf{x}, \mathbf{y})$ is a random Green's function that satisfies the equations

$$
\begin{aligned}
& M g(\mathbf{x}, \mathbf{y})=\delta(\mathbf{x}-\mathbf{y}), \quad \mathbf{x}, \mathbf{y} \in \Omega \\
& g(\mathbf{x}, \mathbf{y})=0, \mathbf{x} \in \partial \Omega, \mathbf{y} \in \Omega
\end{aligned}
$$

Now we can write the unique solution of Equations (2.3) and (2.4) in a simple symbolic form:

$$
u^{\prime}=L f, \quad L=\int_{\Omega} g(\mathbf{x}, \mathbf{y})(\bullet) d y^{d}
$$

If we apply the random operator $M$ to both sides of the equation (2.9) and compare the result with Equation (2.5), we have $M L=\mathrm{I}^{(f)}$, where $\mathrm{I}^{(f)}$ is the identity mapping of the space of the nonrandom functions $f$. If, on the other hand, we apply the random operator $L$ to both sides of Equation (2.5) and compare the result with Equation (2.9), we have $L M=\mathrm{I}^{\left(u^{\prime}\right)}$, where $\mathrm{I}^{\left(u^{\prime}\right)}$ is the identity mapping for the space of the random functions $u^{\prime}$. Thus the random operators $M$ and $L$ are noncommutative because their products-the identity operators $\mathrm{I}^{(f)}$ and $\mathrm{I}^{\left(u^{\prime}\right)}$ operate in different functional spaces.

So, averaging Equation (2.9) over the ensemble of realization $\boldsymbol{\sigma}(\mathbf{x})$, we have for mean field

$$
\begin{aligned}
& U^{\prime}=\left\langle u^{\prime}\right\rangle=U-u_{0} \\
& U^{\prime}=\langle L\rangle f,\langle L\rangle=\int_{\Omega}\langle g(\mathbf{x}, \mathbf{y})\rangle(\square) d y^{d}
\end{aligned}
$$


Because averaging over the probability is related to summation, the nonrandom scalar operator $\langle L\rangle$ exists and is nonsingular. Therefore the unique inverse scalar operator $\langle L\rangle^{-1}$ exists and, when applied to Equation (2.10), we have for $\mathbf{x} \in \Omega$ the averaged equation for $U$

$$
\mathbf{L}_{*}\left(U-u_{0}\right)=f, \mathbf{L}_{*}=\langle L\rangle^{-1}
$$

and for $\mathbf{x} \in \partial \Omega$ the boundary condition

$$
U_{\partial \Omega}=u_{0}
$$

The so-called effective nonrandom scalar operator $\mathbf{L}_{*}$ in the general case is nonlocal and unique. It easy to show that nonrandom operators $\langle L\rangle$ and $\mathbf{L}_{*}=\langle L\rangle^{-1}$ are noncommutative because $\langle L\rangle \mathbf{L}_{*}=\mathrm{I}^{\left(U^{\prime}\right)}$ and $\mathbf{L}_{*}\langle L\rangle=\mathrm{I}^{(f)}$.

Comparing Equations (2.11) and (2.9) we obtain the relationship between the random field $\left(u-u_{0}\right)$ and the nonrandom field $\left(U-u_{0}\right)$

$u-u_{0}=N\left(U-u_{0}\right)$

Here the nonlocal random operator $N$ is the random nonlocal operator $L$ which is normalized by the nonlocal and nonrandom operator $\mathbf{L}_{*}^{-1}$

$N=L \mathbf{L}_{*}$

It is clear that $N$ is a dimensionless linear random operator that for almost all realizations of random tensor-function $\boldsymbol{\sigma}(\mathbf{x})$ maps the uniform space of non-random functions $\left(U-u_{0}\right)$ into the spaces of the relevant realizations of the random functions $\left(u-u_{0}\right)$. In contrast the random operator $N^{-1}=\mathbf{L}_{*}^{-1} L^{-1}=\langle L\rangle L^{-1}$ maps almost all the spaces of relevant realizations of random function $\left(u-u_{0}\right)$ into the uniform space of the nonrandom function $\left(U-u_{0}\right)$. It is evident from (2.12) or (2.13) that the mean operator $\langle N\rangle=\mathrm{I}^{\left(U-u_{0}\right)}$ is the identity mapping of the space of the nonrandom functions $\left(U-u_{0}\right)$.

As we outlined before, now we will find the general relation between the mean fields $U$ and the mean velocity $\mathbf{V}(\mathbf{x})=\langle\mathbf{v}(\mathbf{x})\rangle$. Applying the random vector-operator $-\boldsymbol{\sigma}(\mathbf{x}) \nabla_{x}$ to both sides of Equation (2.13), we can describe the random local velocity vector

$\mathbf{v}(\mathbf{x})=-\boldsymbol{\sigma}(\mathbf{x}) \nabla_{x} u^{\prime}(\mathbf{x})=-\boldsymbol{\sigma}(\mathbf{x}) \nabla_{x}\left[N U^{\prime}\right]$

Or after applying the rule of operator multiplication, and bearing in mind that except for the case of homogenization limit the scalar field $U^{\prime}$ in Equation (2.15) is independent of variable $\mathrm{x}$, we have

$$
\mathbf{v}=\overline{\boldsymbol{\pi}}\left(U-u_{0}\right), \overline{\boldsymbol{\pi}}=-\boldsymbol{\sigma} \nabla N
$$

The random operator $\overline{\boldsymbol{\pi}}$ is the composition of the random operators $-\boldsymbol{\sigma} \nabla$ and $N$ and maps the space of the nonrandom functions $\left(U-u_{0}\right)$ into the space of the random vectors $\mathbf{v}$.

Averaging now Equations (2.16) for the random vectors $\mathbf{v}$ and $\overline{\boldsymbol{\pi}}$, we derive the mean nonrandom velocity vectors

$$
\mathbf{V}=\overline{\mathbf{\Pi}}\left(U-u_{0}\right), \overline{\boldsymbol{\Pi}}=\langle\overline{\boldsymbol{\pi}}\rangle=-\langle\boldsymbol{\sigma} \nabla N\rangle
$$

It is obvious that the compositions-the vector random operator $\overline{\boldsymbol{\pi}}$ and the nonrandom $\overline{\boldsymbol{\Pi}}-$ are nonlocal and they map the nonrandom scalar field $\left(U-u_{0}\right)$ into the vector fields $\mathbf{v}$ and $\mathbf{V}$ 
respectively. Moreover from the condition $\nabla \mathbf{v}=\nabla \mathbf{V}=f$ and Equations (2.16), (2.17) and (2.11) we have the relations: $\nabla \overline{\boldsymbol{\Pi}}=\nabla \overline{\boldsymbol{\pi}}=\mathbf{L}_{*}$ and respectively

$$
\begin{aligned}
& \nabla \mathbf{v}=\mathbf{L}_{*}\left(U-u_{0}\right)=f \\
& \nabla \mathbf{V}=\mathbf{L}_{*}\left(U-u_{0}\right)=f
\end{aligned}
$$

Thus we have a closed system for the local averaged fields, $U$ and $\mathbf{V}$ : Equations (2.11) and (2.12) for the averaged scalar field $U$ and Equations (2.17) for the averaged vector field $\mathbf{V}$. In addition we have obtained the relationships for the local scalar field $u$ and the vector velocity $\mathbf{v}$ in terms of the averaged fields: (2.13) for $u$, and (2.16) for $\mathbf{v}$.

Clearly, all the operators $L, \mathbf{L}_{*}, \boldsymbol{\pi}$ and $\overline{\mathbf{\Pi}}$ are linear. They do not depend on the function $f$,but in general they are related to the domain $\Omega$.

Now, using the operator approach we will partly analyze the case in which the 3 -D domain $\Omega$ is unbounded and the random field $\boldsymbol{\sigma}(\mathbf{x})$ is stochastically homogeneous. This case was studied in Shvidler and Karasaki (2008) with a different approach and here we will compare some results from the two approaches.

As we emphasized in the previous paper (Shvidler and Karasaki, 2008) in this case the local operator $M$ is a self-adjoint, and therefore, Green's function $g(\mathbf{x}, \mathbf{y})$ is symmetric by argument (Courant, 1962): $g(\mathbf{x}, \mathbf{y})=g(\mathbf{y}, \mathbf{x})$, as is the mean Green's function, $G(\mathbf{x}, \mathbf{y})=G(\mathbf{y}, \mathbf{x})$. Because the random field $\boldsymbol{\sigma}(\mathbf{x})$ is stochastically homogeneous $G(\mathbf{x}-\mathbf{y})=G(\mathbf{y}-\mathbf{x})$ and $G$ is a real and even function.

Averaging now the equation (2.9) we have $U^{\prime}(\mathbf{x})$ as the integral in full 3-D space

$$
U^{\prime}(\mathbf{x})=\int G(\mathbf{x}-\mathbf{y}) f(\mathbf{y}) d y^{3}
$$

As in Shvidler and Karasaki (2008), we apply to (2.15) the generalized Fourier transformation (Schwartz, 1961; Yosida, 1978) and obtain the averaged equation in k-space

$$
\bar{F}(\mathbf{k}) \bar{U}^{\prime}(\mathbf{k})=f(\mathbf{k}), \bar{F}(\mathbf{k})=\bar{G}^{-1}(\mathbf{k})
$$

Respectively in the real $\mathrm{x}$-space the averaged nonlocal equation for $U^{\prime}(\mathbf{x})$ is

$$
\int F(\mathbf{x}-\mathbf{y}) U^{\prime}(\mathbf{y}) d y^{3}=f(\mathbf{x})
$$

Substituting now $f(\mathbf{x})$ from Equation (2.17) into Equation (2.9) we have the relationship between the random function $u^{\prime}$ and the nonrandom function $U^{\prime}$ in the explicit form

$$
u^{\prime}(\mathbf{x})=\iint g(\mathbf{x}, \mathbf{y}) F(\mathbf{y}-\mathbf{z}) U^{\prime}(\mathbf{z}) d y^{3} d z^{3}
$$

Therefore the random operator $N$ from equation (2.13) is

$$
N=\iint g(\mathbf{x}, \mathbf{y}) F(\mathbf{y}-\mathbf{z})(\bullet) d y^{3} d z^{3}
$$

and from (2.18) and (2.16) we can find the explicit form of operator $\overline{\mathbf{\Pi}}(\mathbf{x})$ 
$\overline{\mathbf{\Pi}}(\mathbf{x})=\iint \boldsymbol{\Gamma}(\mathbf{x}-\mathbf{y}) F(\mathbf{y}) d y^{3}$

Here, $\boldsymbol{\Gamma}(\mathbf{x}-\mathbf{y})=-\left\langle\boldsymbol{\sigma}(\mathbf{x}) \nabla_{x} g(\mathbf{x}, \mathbf{y})\right\rangle$ is the averaged Green's velocity vector. In the Fourier space Equation (2.20) appears as

$$
\overline{\mathbf{\Pi}}(\mathbf{k})=\overline{\boldsymbol{\Gamma}}(\mathbf{k}) \bar{F}(\mathbf{k})
$$

Multiplay now both parts of equation (2.21) by the function $\bar{U}^{\prime}(\mathbf{k})=\bar{G}(\mathbf{k}) \bar{f}(\mathbf{k})$ and taking into account that $\bar{F}(\mathbf{k}) \bar{G}(\mathbf{k})=1$ we can obtain $\bar{\Pi}(\mathbf{k}) \bar{U}(\mathbf{k})=\overline{\boldsymbol{\Gamma}}(\mathbf{k}) \bar{f}(\mathbf{k})=\overline{\mathbf{V}}(\mathbf{k})$ and in real space we have

$$
\mathbf{V}(\mathbf{x})=\int \boldsymbol{\Pi}(\mathbf{x}-\mathbf{y}) U^{\prime}(\mathbf{y}) d y^{3}
$$

Using now the averaged first equation from (2.1) $\nabla \mathbf{V}(\mathbf{x})=f(\mathbf{x})$ and inserting here $\mathbf{V}(\mathbf{x})$ from (2.22) we obtain

$$
\mathbf{L}_{*} U^{\prime}=f, \quad \mathbf{L}_{*}=\int \nabla_{x} \overline{\mathbf{\Pi}}(\mathbf{x}-\mathbf{y})(\bullet) d y^{3}
$$

As we noted in the previous paper (Shvidler and Karasaki, 2008) the kernel-vector $\overline{\boldsymbol{\Pi}}$ is independent of $f(\mathbf{x})$ and is a unique operator that transforms the scalar field $U^{\prime}$ to the corresponding vector field $\mathbf{V}$.

In the limiting cases of very small scales of heterogeneity and a stochastically homogeneous field $\boldsymbol{\sigma}(\mathbf{x})$, the effective operators $\mathbf{L}_{*}$ and $\overline{\boldsymbol{\Pi}}$ are local and have simple explicit forms $\mathbf{L}_{*}=-\nabla_{x} \boldsymbol{\sigma}_{*} \nabla_{y}$, $\overline{\boldsymbol{\Pi}}=-\boldsymbol{\sigma}_{*} \nabla_{y}$, where $\boldsymbol{\sigma}_{*}=$ const is the effective conductivity tensor in the case of a homogenization limit.

If the field $\boldsymbol{\sigma}(\mathbf{x})$ is global isotropic (see Shvidler and Karasaki, 2008) and domain $\Omega$ is 3 dimensional and unbounded, the vectorial non-local operator $\overline{\boldsymbol{\Pi}}=-\langle\boldsymbol{\sigma} \nabla N\rangle$ must be proportional to a unique vector $\nabla$ and therefore have the form $\overline{\boldsymbol{\Pi}}=-\mathbf{B}^{*}(x) \nabla$. Because the operator $N$ is dimensionless, the nonlocal scalar operator $\mathbf{B}^{*}(x)$ has the same dimension as the conductivity $\boldsymbol{\sigma}(\mathbf{x})$. Then, we have from $(2.23)$

$$
\mathbf{V}(\mathbf{x})=-\int B^{*}(|\mathbf{x}-\mathbf{y}|) \nabla_{y} U^{\prime}(\mathbf{y}) d y^{3}
$$

It is possible to show that for the cases of global orthotropic or transversal isotropic symmetry the averaged equations for $\mathbf{V}$ in relevant symmetry kind coordinate system are similar to (2.24), where the diagonal tensor $\mathbf{B}^{*}()$ is a non-local operator with arguments dependent on the relevant invariants of symmetry. It is evident that the tensors $\boldsymbol{\sigma}_{*}$ and $\mathbf{B}^{*}()$ are unique characteristics for the chosen systems.

\section{Nonsteady Transient Flow with Sources and Sinks}


Let us consider a stochastic system of equations for pressure function $u(\mathbf{x}, t)$ and flow-velocity vector-function $\mathbf{v}(\mathbf{x}, t)$ in a single connected heterogeneous $d$-dimensional domain $\Omega$.

$$
\begin{aligned}
& \beta \frac{\partial m(\mathbf{x}, t)}{\partial t}+\nabla \mathbf{v}(\mathbf{x}, t)=f(\mathbf{x}, t) \\
& m(\mathbf{x}, t)=\theta(\mathbf{x}) u(\mathbf{x}, t) \\
& \mathbf{v}(\mathbf{x}, t)=-\boldsymbol{\sigma}(\mathbf{x}) \nabla u(\mathbf{x}, t)
\end{aligned}
$$

Here $\beta=$ const is a nonrandom coefficient of volume deformation of a liquid-core system, $\theta(\mathbf{x})$ and the elliptic tensor $\boldsymbol{\sigma}(\mathbf{x})$ are porosity and conductivity random functions, respectively, and $f(\mathbf{x}, t)$ is the given density of sources and sinks, which is an integrable and compactly supported non-random function or distribution with bounded support. We assume that the pressure $u(\mathbf{x}, t)$ satisfies the initial condition $u(\mathrm{x}, 0)=u_{0}=\mathrm{const}$, if $\mathbf{x} \in \Omega$ and the boundary condition $u(\mathbf{x}, t)=u_{0}$, if $\mathbf{x} \in \partial \Omega$. If domain $\Omega$ is unbounded, we have the condition at infinity $u(\mathbf{x}, t) \rightarrow u_{0}$.

After combining Equations (3.1) (3.3) we have a parabolic differential equation for the displaced pressure $u^{\prime}(\mathbf{x}, t)=u(\mathbf{x}, t)-u_{0}$

$$
\beta \theta(\mathbf{x}) \frac{\partial u^{\prime}(\mathbf{x}, t)}{\partial t}-\nabla\left[\boldsymbol{\sigma}(\mathbf{x}) \nabla u^{\prime}(\mathbf{x}, t)\right]=f(\mathbf{x}, t)
$$

Thus we have the initial-boundary problem for the $u^{\prime}(\mathbf{x}, t)$ that vanishes on $\partial \Omega$ and at $t=0$.

The $u^{\prime}(\mathbf{x}, t)$ - the unique solution of Equation (3.4) with homogeneous boundary and initial conditions exists and can be a generalized function. For this solution we have

$$
\tilde{M} u^{\prime}=f
$$

where operator $\tilde{M}$ is

$$
\tilde{M}=\beta \theta(\mathbf{x}) \frac{\partial}{\partial t}-\nabla[\boldsymbol{\sigma}(\mathbf{x}) \nabla]
$$

Therefore, for almost all realizations of the conductivity random tensor fields $\boldsymbol{\sigma}(\mathbf{x})$ and porosity random function $\theta(\mathbf{x})$ the operator $\tilde{M}$ maps the space of the Equation (3.4) random solutions $u^{\prime}(\mathbf{x}, t)$ that vanishes on $\partial \Omega$ and at $t=0$ onto the space of the nonrandom functions $f(\mathbf{x}, t)$.

Since the generalized solution of Equation (3.4) with the cited uniform initial and boundary conditions exist and is unique, according Green`s formula we can write

$$
u^{\prime}(\mathbf{x}, t)=\int_{0}^{t} \int_{\Omega} \tilde{g}(\mathbf{x}, t ; \mathbf{y}, \tau) f(y, \tau) d y^{d} d \tau
$$


Here the kernel $\tilde{g}(\mathbf{x}, t ; \mathbf{y}, \tau)$ is the random Green`s function that satisfies the equation

$$
\tilde{M} \tilde{g}(\mathbf{x}, t ; \mathbf{y}, \tau)=\delta(\mathbf{x}-\mathbf{y}) \delta(t-\tau) \quad, \mathbf{x}, \mathbf{y} \in \Omega
$$

under the following boundary and initial conditions, respectively: $\tilde{g}(\mathbf{x}, t ; \mathbf{y}, \tau)=0, \mathbf{x} \in \partial \Omega, \mathbf{y} \in \Omega$, and $\tilde{g}(\mathbf{x}, t ; \mathbf{y}, \tau)=0, t=0$.

Now we can write the solution of equation (3.4) that satisfies the uniform initial and boundary conditions in the form

$$
u^{\prime}=\tilde{L} f, \quad \tilde{L}=\int_{0}^{t} \int_{\Omega} \tilde{g}(\mathbf{x}, t ; \mathbf{y}, \tau)(\cdot) d y^{d} d \tau
$$

As in Section 2, it is easy to show that $\tilde{M} \tilde{L}=\mathrm{I}^{(f)}$ and $\tilde{L} \tilde{M}=\mathrm{I}^{\left(u^{\prime}\right)}$, where $\mathrm{I}^{(f)}$ and $\mathrm{I}^{\left(u^{\prime}\right)}$ are identity mappings in $f$ and $u^{\prime}$ functional spaces, respectively. Thus the random operators $\tilde{L}$ and $\tilde{M}$ are noncommutative.

So, averaging Equations (3.9) of the ensemble of realizations $\boldsymbol{\sigma}(\mathbf{x})$ and $\theta(\mathbf{x})$, we have for the mean displaced pressure $U^{\prime}=\left\langle u^{\prime}\right\rangle$

$$
U^{\prime}=\langle\tilde{L}\rangle f,\langle\tilde{L}\rangle=\int_{0}^{t} \int_{\Omega} \tilde{G}(\mathbf{x}, t ; \mathbf{y} \tau)(\bullet) d y^{d} d \tau
$$

Multiplying both sides of Equation (3.10) by the unique operator $\tilde{\mathbf{L}}_{*}=\langle\tilde{L}\rangle^{-1}$, which in the general case is nonlocal, we derive, for the mean field $U$, the averaged functional equation

$$
\tilde{\mathbf{L}}_{*} U^{\prime}=f \quad, \quad \tilde{\mathbf{L}}_{*}=\langle\tilde{L}\rangle^{-1}
$$

with relevant boundary and initial conditions

$U_{\partial \Omega}^{\prime}=0 \quad, U_{t=0}^{\prime}=0$

By comparing Equations (3.11) and (3.9) we obtain the relationship between the spaces of random field $u^{\prime}(\mathbf{x}, t)$ and the nonrandom field $U^{\prime}$

$u^{\prime}(\mathbf{x})=\tilde{N} U^{\prime}, \tilde{N}=\tilde{L} \tilde{\mathbf{L}}_{*}$

and between the random velocity vector $\mathbf{v}(\mathbf{x}, t)$ and nonrandom field $U^{\prime}$

$$
\mathbf{v}(\mathbf{x}, t)=\tilde{\boldsymbol{\pi}}\left(U-u_{0}\right), \tilde{\boldsymbol{\pi}}=-\boldsymbol{\sigma}(\mathbf{x}) \nabla_{x} \tilde{N}
$$

Note that because the operators $N$ and $\tilde{N}$ are different, the operator $\tilde{\boldsymbol{\pi}}$ is essentially different from the operator $\boldsymbol{\pi}$, which is defined in (2.16). But, of course, as above in Section 2, we are bearing in mind that except at the homogenization limit the local function $U^{\prime}$ in Equation (3.13) is independent from variable $\mathbf{x}$. For this reason the random flow velocity is the product of random nonlocal operator $\tilde{\boldsymbol{\pi}}$ and field $U^{\prime}$. 
Averaging as above Equations (3.14) we can write for the mean velocity vector $\mathbf{V}(\mathbf{x}, t)=\langle\mathbf{v}(\mathbf{x}, t)\rangle$ $\mathbf{V}(\mathbf{x}, t)=\tilde{\mathbf{\Pi}}\left(U-u_{o}\right), \tilde{\boldsymbol{\Pi}}=\langle\tilde{\boldsymbol{\pi}}\rangle=-\left\langle\boldsymbol{\sigma}(\mathbf{x}) \nabla_{x} \tilde{N}\right\rangle$

Using formulas (3.2) and (3.13) we have for $M(\mathbf{x}, t)=\langle m(\mathbf{x}, t)\rangle$

$\mathrm{M}(\mathbf{x}, t)=\mathrm{M}_{0}(\mathbf{x})+\mathrm{M}_{*}(\mathbf{x}, t)$

$\mathrm{M}_{0}(\mathbf{x})=\langle\theta(\mathbf{x})\rangle u_{0}, \quad \mathrm{M}_{*}(\mathbf{x}, t)=\theta_{*}\left[U-u_{0}\right], \theta_{*}=\langle\theta(\mathbf{x}) \tilde{N}\rangle$

It should be noted that unlike the local random function $\theta(\mathbf{x})$, the nonrandom operator $\theta_{*}$ is a nonlocal functional of $\mathbf{x}$-space and time $t$. We will call it- the transient flow effective porosity operator.

Returning now to Equation (3.1) and averaging it, using in addition (3.15) (3.16), and (3.17), and taking into account that the function $\mathrm{M}_{0}$ doesn't depend on $t$, we have the functional equation for the mean function $U(\mathbf{x}, t)$, which satisfy the initial and boundary conditions: $U(\mathbf{x}, 0)=u_{0}, U_{\partial \Omega}(\mathbf{x}, t)=u_{0}$

$\tilde{\mathbf{L}}_{*}\left[U-u_{0}\right]=f, \tilde{\mathbf{L}}_{*}=\beta \frac{\partial}{\partial t} \theta_{*}+\nabla \tilde{\mathbf{\Pi}}$

Clearly, Equations (3.18) generalizes similar conditions for steady-state flow similar to those noted in Section 2.

As emphasized earlier we analyzed the above stated problems in essentially general settings. Now we will consider nonsteady transient flow in the 3-D unbounded domain $\Omega$. We assume that the random conductivity $\boldsymbol{\sigma}(\mathbf{x})$ and porosity $\theta(\mathbf{x})$ fields are stochastically homogeneous in $\Omega$. We assume that the basic local equations (3.1)-(3.4) are valid for any $t>0$ in $\Omega$ and at $|\mathbf{x}| \rightarrow \infty$ the $u(\mathbf{x}, t) \rightarrow u_{0}=$ const . The initial condition is $u(\mathbf{x}, 0)=u_{0}$, for $\mathbf{x} \in \Omega$.

In this case the solution to Equation (3.4) vanishes at infinity and at $t=0$, the reduced pressure, $u^{\prime}(\mathbf{x}, t)$, is valid in $\Omega$.

We introduce the random Green's functuion $\tilde{g}(\mathbf{x}, t ; \mathbf{y}, \tau)$ which satisfies the equation $\tilde{M} \tilde{g}(\mathbf{x}, t ; \mathbf{y}, \tau)=\delta(\mathbf{x}-\mathbf{y}) \delta(t-\tau)$ and, like $u^{\prime}(\mathbf{x}, t)$, is uniform at infinity and $t=0$. In this case

functions $\tilde{\gamma}(\mathbf{x}, t ; \mathbf{y}, \tau)=-\boldsymbol{\sigma}(\mathbf{x}) \nabla_{x} \tilde{g}(\mathbf{x}, t ; \mathbf{y}, \tau)$ and $p(\mathbf{x}, t ; \mathbf{y}, \tau)=\theta(\mathbf{x}) \tilde{g}(\mathbf{x}, t ; \mathbf{y}, \tau)$ that all vanish at $x \rightarrow \infty$ and $t=0$ satisfy the equation :

$\beta \frac{\partial p(\mathbf{x}, t ; \mathbf{y}, \tau)}{\partial t}+\nabla_{x} \tilde{\gamma}(\mathbf{x}, t ; \mathbf{y}, \tau)=\delta(\mathbf{x}-\mathbf{y}) \delta(t-\tau)$

Let us introduce the averaged over ensemble of stochastically homogeneous random fields $\theta(\mathbf{x})$ and $\boldsymbol{\sigma}(\mathbf{x})$, the mean functions $\tilde{G}, P$ and $\tilde{\boldsymbol{\Gamma}}$, which depend on $\mathbf{x}-\mathbf{y}$ and $t-\tau$ 
$\tilde{G}(\mathbf{x}-\mathbf{y}, t-\tau)=\langle\tilde{g}(\mathbf{x}, t ; \mathbf{y}, \tau)\rangle$

$P(\mathbf{x}-\mathbf{y} ; t-\tau)=\langle p(\mathbf{x}, t ; \mathbf{y}, \tau)\rangle$

$\tilde{\boldsymbol{\Gamma}}(\mathbf{x}-\mathbf{y}, t-\tau)=\langle\tilde{\gamma}(\mathbf{x}, t ; \mathbf{y}, \tau)\rangle$

Averaging Equation (3.19) yields

$\beta \frac{\partial \mathrm{P}(\mathbf{x}-\mathbf{y}, t-\tau)}{\partial t}+\nabla_{x} \tilde{\boldsymbol{\Gamma}}(\mathbf{x}-\mathbf{y}, t-\tau)=\delta(\mathbf{x}-\mathbf{y}) \delta(t-\tau)$

We now consider $\bar{T}_{F L}$ and $\bar{T}_{F L}^{-1}$, the direct and inverse Fourier-Laplace generalized transforms (see, for example Schwartz (1961) and Yosida (1978)) in $\mathbf{k}$ and $\mu$ spaces using the following designations

$\overline{\tilde{G}}(\mathbf{k}, \mu)=\bar{T}_{F L} \tilde{G}, \quad \overline{\mathrm{P}}(\mathbf{k}, \mu)=\bar{T}_{F L} \mathrm{P}, \quad \overline{\tilde{\Gamma}}(\mathbf{k}, \mu)=\bar{T}_{F L} \tilde{\boldsymbol{\Gamma}}$

and we introduce the following vector $\overline{\boldsymbol{\Pi}}_{\otimes}(\mathbf{k}, \mu)$ and scalar function $\bar{S}(\mathbf{k}, \mu)$

$\overline{\boldsymbol{\Pi}}_{\otimes}(\mathbf{k}, \mu)=\overline{\tilde{\boldsymbol{\Gamma}}}(\mathbf{k}, \mu) \overline{\tilde{G}}^{-1}(\mathbf{k}, \mu), \quad \bar{S}(\mathbf{k}, \mu)=\overline{\mathrm{P}}(\mathbf{k}, \mu) \overline{\tilde{G}}^{-1}(\mathbf{k}, \mu)$

It is straightforward to show that applying the transform $\bar{T}_{F L}$ to Equation (3.23), we have in Fourier and Laplace space:

$\mu \beta \bar{S}(\mathbf{k}, \mu) \overline{\tilde{G}}(\mathbf{k}, \mu)+2 \pi i \mathbf{k} \bar{\Pi}_{\otimes}(\mathbf{k}, \mu) \overline{\tilde{G}}(\mathbf{k}, \mu)=1$

Multiplying Equation (3.26) by $\bar{f}(\mathbf{k}, \mu)=\bar{T}_{F L} f(\mathbf{x}, t)$ and using the following formulas $\bar{U}^{\prime}(\mathbf{k}, \mu)=\overline{\tilde{G}}(\mathbf{k}, \mu) \bar{f}(\mathbf{k}, \mu)$

$\overline{\mathbf{V}}(\mathbf{k}, \mu)=\overline{\boldsymbol{\Pi}}_{\otimes}(\mathbf{k}, \mu) \overline{\tilde{G}}(\mathbf{k}, \mu) \bar{f}(\mathbf{k}, \mu)=\overline{\mathbf{\Pi}}_{\otimes}(\mathbf{k}, \mu) \bar{U}^{\prime}(\mathbf{k}, \mu)$

$\bar{M}^{\prime}(\mathbf{k}, \mu)=\bar{S}(\mathbf{k}, \mu) \bar{U}^{\prime}(\mathbf{k}, \mu)$

We obtain the general averaged equation in Fourier-Laplace space:

$\mu \beta \overline{\mathrm{M}}^{\prime}(\mathbf{k}, \mu)+2 \pi i \mathbf{k} \overline{\mathbf{V}}(\mathbf{k}, \mu)=\bar{f}(\mathbf{k}, \mu)$

Clearly, for any $\mathbf{x}$ and $(t-\tau)$ in stochastically homogeneous fields $\boldsymbol{\sigma}(\mathbf{x})$ and $\theta(\mathbf{x})$ the kernelvector $\Pi_{\otimes}(\mathbf{x}-\mathbf{y}, t-\tau)$ is an odd vector function in $\mathbf{x}-\mathbf{y}$ space. On the other hand the transformed moment $\bar{P}(\mathbf{k}, \mu)$ and Green`s function $\overline{\tilde{G}}(\mathbf{k}, \mu)$ are even function in $\mathbf{k}$-space and for this reason the functions $\bar{S}(\mathbf{k}, \mu)$ and $S(\mathbf{x}-\mathbf{y}, t-\tau)$ are even in the same way. Taking this into account the averaged nonlocal system for scalar and vector mean functions $U$ and $\mathbf{V}$ is:

$\beta \frac{\partial \mathrm{M}^{\prime}(\mathbf{x}, t)}{\partial t}+\nabla \mathbf{V}(\mathbf{x}, t)=f(\mathbf{x}, t)$ 


$$
\begin{aligned}
& \mathbf{M}^{\prime}(\mathbf{x}, t)=\int_{0}^{t} \int S(\mathbf{x}-\mathbf{y}, t-\tau)\left[U(\mathbf{y}, \tau)-u_{0}\right] d y^{3} d \tau \\
& \mathbf{V}(\mathbf{x}, t)=\int_{0}^{t} \int \Pi_{\otimes}(\mathbf{x}-\mathbf{y}, t-\tau)\left[U(\mathbf{y}, \tau)-u_{0}\right] d y^{3} d \tau \\
& U(\mathbf{x}, 0)=U(\infty, t)=u_{0}
\end{aligned}
$$

Or one nonlocal equation for $U^{\prime}$

$$
\beta \frac{\partial}{\partial t} \int_{0}^{t} \int S(\mathbf{x}-\mathbf{y}, t-\tau) U^{\prime}(\mathbf{y}, \tau) d y^{3} d \tau+\nabla_{x} \int_{0}^{t} \int_{0} \boldsymbol{\Pi}_{\otimes}(\mathbf{x}-\mathbf{y}, t-\tau) U^{\prime}(\mathbf{y}, \tau) d y^{3} d \tau=f(\mathbf{x}, t)
$$

\section{Nonreactive Solute Transport}

Here we consider the stochastic transport of nonreactive solute. As before we assume that the conductivity and porosity are random fields and that therefore flow velocity and solute concentration are random fields as well.

In the conventional approach to studying these similar processes the random flow velocity vector field is considered as given and usually as stochastically homogeneous. In addition the initial distribution of the solute is assumed to be a nonrandom plume.

Here, however, we will consider both processes (flow and transport) jointly. We will assume that at the initial moment in the field the solute is absent, but that later, solute is input in the field and output from the field through distributed sources or sinks.

It is evident that depending on the method used to input solute in the field it may be injected with some volumes of carrier liquid. If these volumes are relatively significant they can reasonably be included in the flow balance equation. If the solute is output from the system together with liquid, we must account for it, because the given or prescribed density of liquid sinks and solute sinks are mutually dependent, for example, proportional.

Now we consider flow and transport in the three-dimensional unbounded domain $\Omega$. The local transport equations are as follows:

$$
\begin{aligned}
& \frac{\partial a(\mathbf{x}, t)}{\partial t}+\nabla \mathbf{q}(\mathbf{x}, t)=\varphi(\mathbf{x}, t) \\
& a(\mathbf{x}, t)=\theta(\mathbf{x}) c(\mathbf{x}, t) \\
& \mathbf{q}(\mathbf{x}, t)=\mathbf{v}(\mathbf{x}, t) c(\mathbf{x}, t)-\mathbf{D} \nabla c(\mathbf{x}, t) \\
& c(\mathbf{x}, 0)=0
\end{aligned}
$$

The function $c(\mathbf{x}, t)$ is the concentration of nonreactive solute that vanishes at infinity. We assume that the conductivity tensor $\boldsymbol{\sigma}(\mathbf{x})$ and porosity function $\theta(\mathbf{x})$ are random functions, $\mathbf{D}$ is a constant and nonrandom positive definite dispersion tensor and therefore the vectors, the flow- 
velocity $\mathbf{v}(\mathbf{x}, t)$ and the solute flux $\mathbf{q}(\mathbf{x}, t)$, as well as the scalar-functions, $c(\mathbf{x}, t)$ and $a(\mathbf{x}, t)$ are random. We assume that the function $\varphi(\mathbf{x}, t)$ is nonrandom solute source's density. As we note later for this case the density of liquid sinks $f(\mathbf{x}, t)$ and $\varphi(\mathbf{x}, t)$ must be independent. To close the system (4.1)-(4.4) we will use additional subsystem of equations $(1,3)-(3,3)$ which describe the field $\mathbf{v}(\mathbf{x}, t)$.

Let us assume that the initial value of pressure $u(\mathbf{x}, 0)=u_{0}=$ const and at infinity $u(\mathbf{x}, t)=u_{0}$ as $x \rightarrow \infty$. Combining Equations (4.1)-(4.3) and (3.14) we can write for $c(\mathbf{x}, t)$ the linear parabolic equation and the initial and boundary conditions as follows:

$$
\begin{aligned}
& \hat{M} c(\mathbf{x}, t)=\varphi(\mathbf{x}, t), \hat{M}=\theta(\mathbf{x}) \frac{\partial}{\partial t}+\nabla \tilde{\pi}\left(U-u_{0}\right)-\nabla D \nabla \\
& c(\mathbf{x}, 0)=0, c(\mathbf{x}, t)=0 \text { if } x \rightarrow \infty
\end{aligned}
$$

Introducing Green's function $g_{c}(\mathbf{x}, t ; \mathbf{y}, \tau)$ that satisfies the equation $\hat{M} g_{c}=\delta(\mathbf{x}-\mathbf{y}) \delta(t-\tau)$ with a uniform initial and boundary conditions we can write Green's formula relationship as

$c(\mathbf{x}, t)=\int_{0}^{t} \int_{\Omega} g_{c}(\mathbf{x}, t ; \mathbf{y}, \tau) \varphi(\mathbf{y}, \tau) d y^{3} d \tau$

Defining the operator $L_{c}=\int_{0}^{t} \int_{\Omega} g_{c}(\mathbf{x}, \mathbf{y} ; t, \tau)(\square) d y^{3} d \tau$ we can write Equation (4.7) in the form $c=L_{c} \varphi$

and by averaging both sides of the last equation over the ensemble random fields $\boldsymbol{\sigma}(\mathbf{x})$ and $\theta(\mathbf{x})$ and designating $C(\mathbf{x}, t)=\langle c(\mathbf{x}, t)\rangle$ and $G_{c}(\mathbf{x}, t ; \mathbf{y}, \tau)=\langle g(\mathbf{x}, t ; \mathbf{y}, \tau)\rangle$, we have

$$
C=\left\langle L_{c}\right\rangle \varphi
$$

Multiplying both sides of Equation (4.9) by the unique inverse operator $\left\langle L_{c}\right\rangle^{-1}$ leads to the basic equation for mean concentration $C$

$$
\mathbf{L}_{c}^{*} C=\varphi, \mathbf{L}_{c}^{*}=\left\langle L_{c}\right\rangle^{-1}
$$

The unique operator $\mathbf{L}_{c}^{*}$ we shall call as effective.

From Equations (4.8) and (4.10) we have the relationship between the random and mean concentration

$$
c=N_{c} C, N_{c}=L_{c} \mathbf{L}_{c}^{*}
$$

The scalar random operator $N_{c}$ for almost all realization of the random fields $\boldsymbol{\sigma}(\mathbf{x})$ and $\theta(\mathbf{x})$ maps the uniform nonrandom function space $C$ into relevant realizations of random 
functions space $c$. Evidently we have $\left\langle N_{c}\right\rangle=\mathrm{I}^{(C)}$ - identity mapping in the space of nonrandom functions $C$.

Using these results and the relation (4.3), we can find the mean flux $Q$

$$
Q=\langle q\rangle=\langle\mathbf{v} c-\mathbf{D} \nabla c\rangle=\left\langle\tilde{\boldsymbol{\pi}}\left(U-u_{0}\right) N_{c} C-\mathbf{D} \nabla N_{c} C\right\rangle
$$

And because the mean concentration $C$ is nonrandom and $\left\langle N_{c}\right\rangle=I^{(C)}$, we can write

$$
Q=\mathbf{W} C-\mathbf{D} \nabla C, \quad \mathbf{W}=\left\langle\tilde{\boldsymbol{\pi}}\left(U-u_{0}\right) N_{c}\right\rangle
$$

The vector $\mathbf{W}$ can be called as the effective transport velocity, which reflects the convective, and because $\mathbf{W}$ is linked with $N_{c}$, diffusion mechanisms of transport.

Similarly we obtain $A=\langle a\rangle=\langle\theta c\rangle=\left\langle\theta N_{c} C\right\rangle=\left\langle\theta N_{c}\right\rangle C$ or

$$
A=\theta^{*} C, \quad \theta^{*}=\left\langle\theta N_{c}\right\rangle
$$

The nonrandom functional $\theta^{*}$ can be called as the effective transport porosity. It is evident that both effective functionals $\theta^{*}$ and $\theta_{*}$ from Section 3 , unlike the porous media function $\theta(\mathbf{x})$, also reflect appropriately different processes: transport and transient flow.

Averaging Equation (4.1) and using the formulas (4.13) and (4.14) we can write the equation and initial and boundary conditions for the mean concentration $C(\mathbf{x}, t)$ :

$$
\begin{aligned}
& \frac{\partial}{\partial t}\left[\theta^{*}(\mathbf{x}, t) C(\mathbf{x}, t)\right]+\nabla[\mathbf{W}(x, t) C(\mathbf{x}, t)]-\nabla \mathbf{D} \nabla C(\mathbf{x}, t)=\varphi(\mathbf{x}, t) \\
& C(\mathbf{x}, 0)=0, \text { by } \mathbf{x} \in \Omega \text { and } C(\mathbf{x}, t)=0 \text { if } x \rightarrow \infty
\end{aligned}
$$

From Equations (4.14) and (4.9) we can write the semi-explicit form of the effective operator $\mathbf{L}_{c}^{*}$, which includes the effective operators $\theta^{*}$ and $\mathbf{W}$.

$$
\mathbf{L}_{c}^{*}=\frac{\partial}{\partial t} \theta^{*}(\mathbf{x}, t)+\nabla \mathbf{W}(\mathbf{x}, t)-\nabla \mathbf{D} \nabla
$$

Finally we will study the somewhat exotic case of nonreactive solute transport in an unbounded porous space with stochastically homogeneous porosity and conductivity, in which (moreover) the flow-velocity is given as a stochastically homogeneous vector field. Of course, this case is admittedly not included in the above-stated general theory. We need to stress that in an unbounded space the stochastically homogeneous velocity field does not exist, but in some cases is an acceptable approximation.

So, returning again to the basic system of equations (4.1)-(4.4), we introduce the random Green's function $\tilde{g}_{c}(\mathbf{x}, t ; \mathbf{y}, \tau)$ that satisfies the equation:

$$
\theta(\mathbf{x}) \frac{\partial \tilde{g}_{c}(\mathbf{x}, t ; \mathbf{y}, \tau)}{\partial t}+\nabla\left[\mathbf{v}(\mathbf{x}, t) \tilde{g}_{c}(\mathbf{x}, t ; \mathbf{y}, \tau)\right]-\nabla\left[\mathbf{D} \nabla \tilde{g}_{c}(\mathbf{x}, t ; \mathbf{y}, \tau)\right]=\delta(\mathbf{x}-\mathbf{y}) \delta(t-\tau)
$$


with $\tilde{g}_{c}$ vanishing at $t=\tau=0$ and at $x \rightarrow \infty$ for any $t$.

If the vector function $\mathbf{v}(\mathbf{x}, t)$ and the scalar function $\theta(\mathbf{x})$ are defined as stochastically homogeneous fields in $\mathbf{x}$-space and stationary in time $\mathrm{t}$, we have the following relationships:

$$
\begin{aligned}
& \left\langle\tilde{g}_{c}(\mathbf{x}, t ; \mathbf{y}, \tau)\right\rangle=\tilde{G}_{c}(\mathbf{x}-\mathbf{y}, t-\tau) \\
& \left\langle\theta(\mathbf{x}) \tilde{g}_{c}(\mathbf{x}, t ; \mathbf{y}, \tau)\right\rangle=H(\mathbf{x}-\mathbf{y}, t-\tau) \\
& \left\langle\mathbf{v}(\mathbf{x}, t) \tilde{g}_{c}(\mathbf{x}, t ; \mathbf{y}, \tau)\right\rangle=\mathbf{F}_{c}(\mathbf{x}-\mathbf{y}, t-\tau)
\end{aligned}
$$

Applying $\bar{T}_{F L}$-the generalized Fourier-Laplace transforms in the vector $\mathbf{k}$ and the scalar $\mu$ space for the functions $\tilde{G}_{c}, H$ and $\mathbf{F}_{c}$, we have

$$
\overline{\tilde{G}}_{c}(\mathbf{k}, \mu)=\bar{T}_{F L} \tilde{G}_{c}(\mathbf{x}-\mathbf{y}, t-\tau), \bar{H}(\mathbf{k}, \mu)=\bar{T}_{F L} H(\mathbf{x}-\mathbf{y}, t-\tau), \overline{\mathbf{F}}_{c}(\mathbf{k}, \mu)=\bar{T}_{F L} \mathbf{F}_{c}(\mathbf{x}-\mathbf{y}, t-\tau)
$$

Introducing functions

$$
\overline{\tilde{\theta}}(\mathbf{k}, \mu)=\bar{H}(\mathbf{k}, \mu) \overline{\tilde{G}}_{c}^{-1}(\mathbf{k}, \mu), \overline{\mathbf{W}}(\mathbf{k}, \mu)=\overline{\mathbf{F}}_{c}(\mathbf{k}, \mu) \overline{\tilde{G}}_{c}(\mathbf{k}, \mu)
$$

it is straightforward to show that averaging Equation (4.18), applying to it the $\bar{T}_{F L}$ transform and using the designations (4.23), we have

$\mu \overline{\tilde{\theta}}(\mathbf{k}, \mu) \overline{\tilde{G}}_{c}(\mathbf{k}, \mu)+2 \pi i \mathbf{k} \overline{\mathbf{W}}(\mathbf{k}, \mu) \overline{\tilde{G}}_{c}(\mathbf{k}, \mu)-4 \pi^{2} \mathbf{k}(\mathbf{D k}) \overline{\tilde{G}}_{c}(\mathbf{k}, \mu)=1$

Multiplying Equation (4.24) by $\bar{\varphi}(\mathbf{k}, \mu)=\bar{T}_{F L} \varphi(\mathbf{x}, t)$ and taking into account that for the $\bar{T}_{F L}$ transform of $C(\mathbf{x}, t)=\langle c(\mathbf{x}, t)\rangle$ we have

$$
\bar{C}(\mathbf{k}, \mu)=\overline{\tilde{G}}_{c}(\mathbf{k}, \mu) \bar{\varphi}(\mathbf{k}, \mu)
$$

and we obtain in $(\mathbf{k}, \mu)$ space the general equation for the transformed mean concentration $\bar{C}(\mathbf{k}, \mu)$

$\mu \bar{\theta}_{*}(\mathbf{k}, \mu) \bar{C}(\mathbf{k}, \mu)+2 \pi i \mathbf{k} \overline{\mathbf{W}}(\mathbf{k}, \mu) \bar{C}(\mathbf{k}, \mu)-4 \pi^{2}(\mathbf{k D k}) \bar{C}(\mathbf{k}, \mu)=\bar{\varphi}(\mathbf{k}, \mu)$

Then, using the inverse transform $\bar{T}_{F L}^{-1}$ on Equation (4.29) and denoting

$$
\theta_{*}(\mathbf{x}, t)=T_{F L}^{-1} \bar{\theta}_{*}(\mathbf{k}, \mu), \mathbf{Q}(\mathbf{x}, t)=\langle q(\mathbf{x}, t)\rangle, \mathbf{W}(\mathbf{x}, t)=\bar{T}_{F L}^{-1} \overline{\mathbf{W}}(\mathbf{k}, \mu)
$$

we obtain the nonlocal equations in space-time for the mean concentration $C(\mathbf{x}, t)$ and for the mean solute flux -vector $\mathbf{Q}(\mathbf{x}, t)$

$$
\frac{\partial A(\mathbf{x}, t)}{\partial t}+\nabla \mathbf{Q}(\mathbf{x}, t)=\varphi(\mathbf{x}, t)
$$




$$
\begin{aligned}
& A(\mathbf{x}, t)=\int_{0}^{t} \int_{*} \theta_{*}(\mathbf{x}-\mathbf{y}, t-\tau) C(\mathbf{y}, \tau) d y^{3} d \tau \\
& \mathbf{Q}(\mathbf{x}, t)=\int_{0}^{t} \int \mathbf{W}(\mathbf{x}-\mathbf{y}, t-\tau) C(\mathbf{y}, \tau) d y^{3} d \tau-\mathbf{D} \nabla C(\mathbf{x}, t) \\
& C(\mathbf{x}, 0)=0
\end{aligned}
$$

\section{Summary}

We have described the general form for the exactly averaged system of basic equations of steady flow, nonsteady transient flow and solute transport in arbitrary random bounded or unbounded domains of any dimension with sources and sinks. We examined the validity of the averaged descriptions and the generalized law for some nonlocal models. The approach described in the present paper does not require assuming the existence of any small parameters, for example, small scales of heterogeneity or small perturbation of conductivity field.

\section{Acknowledgments}

The authors would like to thank Dr. D. Silin of Lawrence Berkeley National Laboratory for constructive comments. This work was conducted under the U.S. Department of Energy Contract No. DE-AC02-05CH11231.

\section{References}

Bakhvalov, N.S., Panasenko, G.P.: Homogenization: Averaging Processes in Periodic Media; Mathematical Problems in the Mechanics of Composite Material. KluverAcademic, Dordrecht(1989)

Courant, R.: Partial Differential Equations. Interscience, New York (1962)

Finkelberg,V.M.:1964, Mean field strength in an inhomogeneous medium, Soviet Physics JETP, vol 19, No.2, 494-498.

Hashin, Z., Shtrikman S.: 1962, On some varitional principles in anisotropic and nonhomogeneous elasticity, J. Mech. Phys. Solids., 10, No.4, 335

Hornung, U. (ed.).: 1997, Homogenization and Porous Media, IAM, Springer-Verlag, Berlin.

Indelman, P., Abramovich B.:1994, Nonlocal properties of nonuniform averaged flows in heterogeneous media, Water Resour.Res., 30 (12),3385-3393.

Indelman, P.: 1996, Averaging of unsteady flows in heterogeneous media of stationary conductivity. J. Fluid Mech., 310, 39-60.

Indelman, P.: 2001, Steady-state source flow in heterogeneous porous media. Transport in Porous Media, 45:105-127.

Indelman, P.: 2002, On mathematical models of average flow in heterogeneous formations. Transport in Porous Media, 48:209-224. 
Koshlyakov, N.S., M.M. Smirnov and E.B.Gliner.: 1964, Differential Equations of Mathematical Physics, North-Holland Publishing Co. Amsterdam.

Neuman, S.P., Orr S.: 1993, Prediction of steady state flow in nonuniform geologic media by conditional moments: exact nonlocal formalism, effective conductivies, and weak approximation, Water Resour. Res., 29 (2), 341-364.

Tartakovsky D.M., and S.P.Neuman : 1998, Transient flow in bounded randomly heterogeneous domains 2. Localization of conditional mean equations and temporal nonlocality effects. Water Resour. Res., 34 (1), 13-20.

Schwartz, L.: 1961, Methods Mathematiques Pour Les Sciences Physiques, Herman \& Cie, Paris.

Shermergor T. D.: 1977, The Elasticity Theory of Microheterogeneous Media. Nauka, Moscow (in Russian)

Shvidler, M.I.:1985, Statistical Hydrodynamics of Porous Media. (In Russian), Nedra, Moscow.

Shvidler, M.I.: 1993, Correlation model of transport in Random Fields. Water Resour. Res., 29 (9), 3189-3199

Shvidler, M., and K.Karasaki.: 1999, Investigation of the exactly averaged equations of flow and transport in random porous media. Abstract, In Transactions, AGU Fall Meeting, San Francisco, 1999.

Shvidler, M., and K. Karasaki: 2001, Exactly Averaged Stochastic Equations for Flow and Transport in Random Media. Earth Sciences Division Ernest Orlando Lawrence Laboratory, University of California, Berkeley, LBNL-49529, Preprint, 22.

Shvidler, M., and K.Karasaki: 2005, Averaging of Stochastic Equations for Flow and Transport in Porous Media. Earth Sciences Division, Ernest Orlando Lawrence Berkeley Laboratory, University of California, Berkeley, LBNL-56935, Preprint, 56.

Shvidler, M., and K.Karasaki: 2008, Exact Averaging of Stochastic Equations for Flow in Porous Media. Transport in Porous Media, 72 : 295-310.

Yosida, K.: 1978, Functional Analysis, Fifth Edition, Springer-Verlag, Berlin.

Zhikov, V.V., S.M. Kozlov, and O.A. Oleinik: 1994, Homogenization of Differential Operators and Integral Functionals, Springer-Verlag, Berlin. 


\section{DISCLAIMER}

This document was prepared as an account of work sponsored by the United States Government. While this document is believed to contain correct information, neither the United States Government nor any agency thereof, nor The Regents of the University of California, nor any of their employees, makes any warranty, express or implied, or assumes any legal responsibility for the accuracy, completeness, or usefulness of any information, apparatus, product, or process disclosed, or represents that its use would not infringe privately owned rights. Reference herein to any specific commercial product, process, or service by its trade name, trademark, manufacturer, or otherwise, does not necessarily constitute or imply its endorsement, recommendation, or favoring by the United States Government or any agency thereof, or The Regents of the University of California. The views and opinions of authors expressed herein do not necessarily state or reflect those of the United States Government or any agency thereof or The Regents of the University of California.

Ernest Orlando Lawrence Berkeley National Laboratory is an equal opportunity employer. 